\title{
Ecuador declarado país libre de la fiebre aftosa. Oportunidades y desafios
}

\section{Ecuador as an aftosa fever free-country. Opportunities and challenges}

\author{
Francisco Gutiérrez León \\ ${ }^{\prime}$ Universidad Central del Ecuador. Facultad de Ciencias Agrícolas. Jerónimo Leiton y Av. La Gasca s/n. Ciudadela Universitaria. 170521 \\ Quito, Ecuador
}

\begin{abstract}
Resumen
Ecuador fue declarado en el 2015 país libre de fiebre aftosa por parte de la Organización Mundial de Sanidad Animal (OIE), requisito indispensable para la exportación de productos lácteos y carne. La producción de leche en el país ha crecido sostenidamente durante la última década. Según datos obtenidos del Instituto Nacional de Estadísticas y Censos (INEC), en el año 2012 se produjeron 5,675,067 litros de leche al día en el país. Por otro lado, el consumo per cápita en el país para el año 2015 es de 100 litros de leche por habitante por año, según datos recabados de la Federación Panamericana de Lechería (FEPALE). Como resultado de esto, se ha creado una brecha entre las fuerzas de mercado oferta y demanda de leche. Desde la perspectiva de la oferta, existe una sobre producción de leche en ciertos meses del año. Por el lado de la demanda, el consumo nacional per cápita es relativamente bajo en comparación al estándar internacional establecido por la FEPALE y la Organización Mundial de la Salud de 150 litros de leche por habitante por año.
\end{abstract}

Palabras clave: libre de fiebre aftosa, consumo de leche, producción de leche.

\begin{abstract}
Ecuador was declared an aftosa fever free-country in 2015 by the World Animal Health Organization. This is a mandatory requirement for exportation purposes of meat and milk products. The milk production in the country has been increasing progresively along the last decade. According to the Instituto Nacional de Estadisticas y Censos (INEC), in the year 2012 the national milk production was 5, 675,067 liters per day. On the other hand, the national milk consumption per inhabitant is 100 liters per year in 2015, data obtained from the Federacion Panamericana de Lecheria (FEPALE). As a result of this, an spread has been created between the milk supply and demand market forces. From the supply perspective, there is an over production of milk in specific months of the year. As for the demand, the national consumption per inhabitant is relatively low comparing to the international standard given by the FEPALE and the World Health Organization of 150 liters per inhabitant per year.
\end{abstract}

Keywords: aftosa fever free, milk consumption, milk production. 


\section{Introducción}

La producción de leche en el mundo es una actividad con un gran dinamismo, países como China, que en el pasado no consumía productos lácteos, ha incrementado considerablemente su demanda, y otros como la India han incursionado en el mercado como productores de leche (Sharma \& Rou, 2014. Existen países que siempre se han caracterizado por la producción y consumo de leche como los Estados Unidos y los miembros de la Unión Europa. En Sudamérica, Brasil pasó, en tan solo dos décadas, de ser un importador de productos lácteos a ser el primer productor de leche en el continente, seguido por Argentina, Colombia, Chile y Uruguay. El mercado de la leche a nivel mundial es muy competitivo y el precio al cual se cotiza los productos lácteos es errático y puede fluctuar súbitamente, Ecuador puede entrar a este mercado gracias a la declaración de país libre de fiebre aftosa.

La ganadería en el Ecuador es una de las principales actividades agropecuarias. La superficie que se destina para pastos cultivados y pastos naturales es de 4,976,122 hectáreas, lo que representa el 41.81\% de la tierra cultivada en Ecuador (INEC, 2012). En el año 2012, la producción de leche en Ecuador alcanzó los 5,675,067 litros al día, siendo la región Sierra la que más aporta en este rubro con un $76.79 \%$, seguido de la Costa con el $15.35 \%$ y el Oriente con el $7.86 \%$. En relación al promedio de litros de leche por vaca producidos, la región que más se destaca es la Sierra con 6.46 litros/vaca, debido a la gran cantidad de ganado lechero presente y a pastos cultivados y naturales que sirven para su alimentación. La región Oriental ocupa el segundo lugar con 4.50 litros/ vaca y por último la región Costa con 3.11 litros/ vaca (INEC, 2012). Estas cifras dejan ver claramente la importancia de la ganadería lechera tanto para la seguridad y soberanía alimentaria como para las economías locales del país.

Una de las grandes limitantes para el desarrollo y expansión del sector lechero en Ecuador ha sido la imposibilidad de exportar productos lácteos debido a la prevalencia de la fiebre aftosa. Este artículo analiza las oportunidades y retos del sector lechero ecuatoriano una vez que Ecuador ha sido declarado país libre de aftosa.

\section{Estatus sanitario en Suramérica}

Ecuador fue declarado libre de fiebre aftosa por parte de la Organización Mundial de Sanidad Animal (OIE). En febrero del 2015 este objetivo se lo logró luego de varios años de campañas de vacunación que se realizaron dos veces en el año a nivel nacional, la institución que coordina estos planes de vacunación en el país es Agrocalidad (MAGAP, 2015). Esta declaración abre las puertas al país para poder exportar leche, carne y sus derivados. También es posible exportar productos de ovinos, caprinos y cerdos, los cuales también pueden padecer esta enfermedad. En Sudamérica casi todos los países han logrado certificase como libres de esta enfermedad a excepción de Venezuela y algunas partes de Brasil (OIE, 2015) (Figura 1).

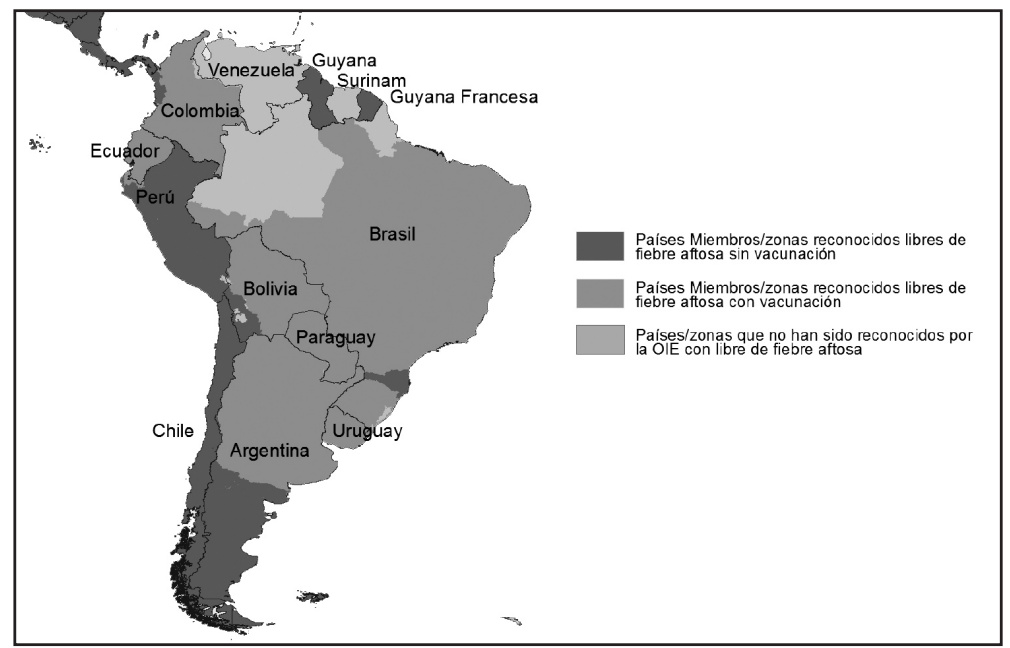

Figura 1. Mapa oficial del estatus de fiebre aftosa en Sudamérica. Fuente: OIE (2015) 


\section{Producción y consumo de leche}

La ganadería de leche bovina en el país ha crecido de manera sostenida durante la última década. Varios productores han visto una alternativa de ingresos en la ganadería de leche debido a que esta ofrece ingresos más estables que los observados para la producción agrícola. Mediante Acuerdo del Ministerio de Agricultura, Ganadería y Pesca se fijó el precio de leche a nivel de finca en US \$ 0.42 por litro de leche cruda (MAGAP, 2013). En contraparte, otros cultivos como, por ejemplo la papa, exhiben precios fluctuantes en el mercado. Por otro lado, los cultivos de la Sierra tienen ciclos vegetativos superiores a los 5 meses lo que dilata el período necesario para la obtención de ingresos. Adicionalmente, la ganadería no requiere uso intensivo de mano de obra a diferencia de los cultivos, los cuales requieren mano de obra en labores como escarda, fertilización, aporque, y la cosecha. Estos factores se ven reflejados en el incremento que ha experimentado la producción de leche durante el período 2005-2012 (INEC, 2012) (Figura 2).

Es necesario recalcar que el aumento de la producción de leche obedece a un incremento de consumo, lo que trae como consecuencia que en ciertas épocas del año, exista sobreproducción. Según la Federación Panamericana de Lechería (FEPALE) el consumo recomendado es entre 150-180 litros de leche/habitante/año, en la región, países como Argentina, Uruguay, Chile y Brasil tienen este consumo (Londinsky, 2015). Ecuador registra un consumo per cápita de 100 litros de leche (Figura 3), lo que refleja que se requieren diseñar y aplicar políticas para incrementar el consumo de leche en Ecuador. Una alternativa es incluir leche en los desayunos escolares de los niños, mientras que el reto más grande es incentivar el consumo de leche entre toda la población.

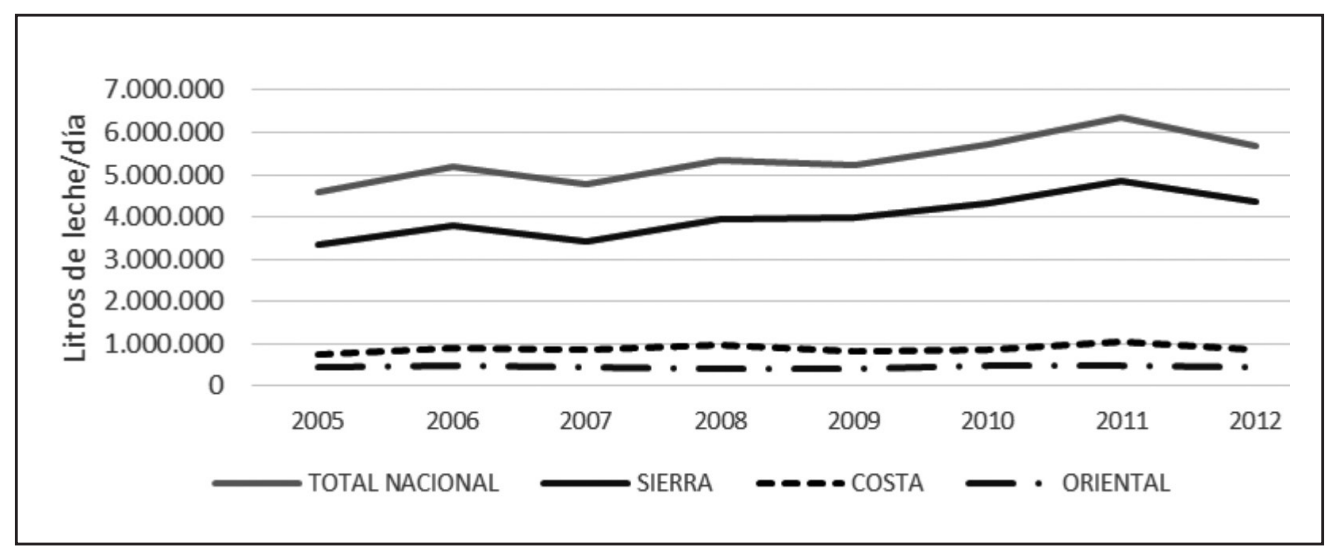

Figura 2. Producción de leche cruda bovina en las tres regiones del Ecuador Fuente: Elaboración propia del autor con datos del INEC (2012)

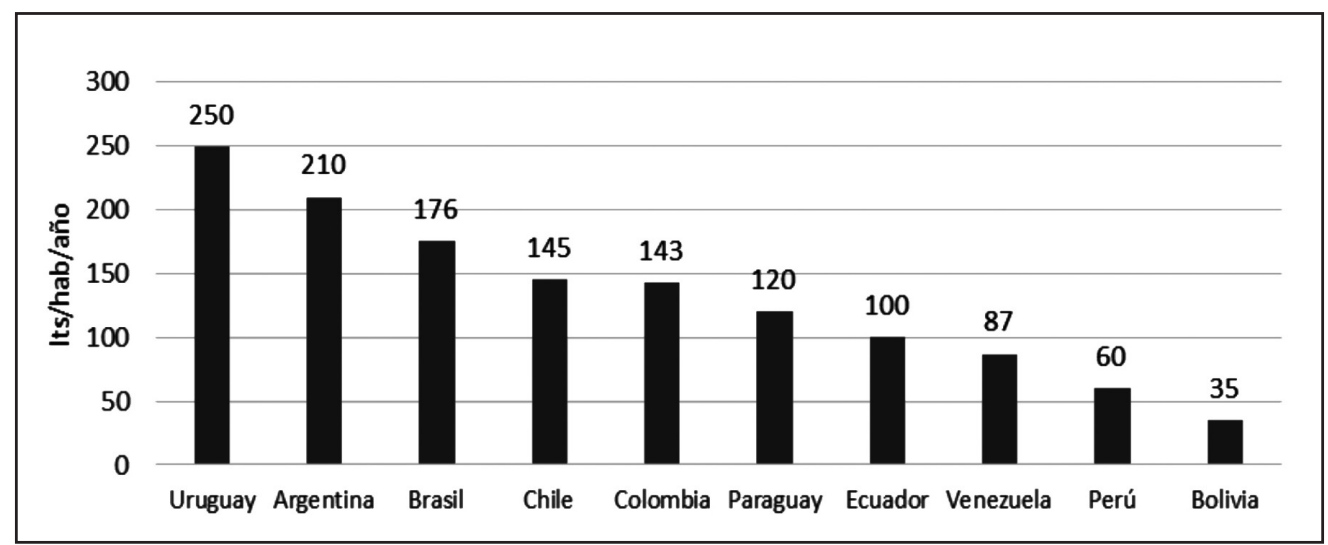

Figura 3. Consumo per cápita de leche litro/habitante/año en Sudamérica. Fuente: Adaptado de la Federación Panamericana de Lechería (FEPALE). 


\section{Mercado internacional}

La exportación de productos lácteos tiene el potencial de convertirse ser una alternativa para nuestros excedentes de leche cruda. Generalmente, estos excedentes de leche se los exporta en forma de leche en polvo, quesos, yogurt, entre otros productos procesados. Sin embargo, una limitante es el precio que se paga por litro de la leche fijado para el mercado local, el cual es uno de los más altos en Sudamérica. Mientras que en el país se paga US \$ 0.42 por litro de leche cruda (Figura 4), países de la región que exportan leche y sus derivados como Uruguay y Argentina pagan entre US \$ 0.36 y US \$ 0.34 respectivamente (FEDEGAN, 2015). Estos valores reflejan que, en las condiciones actuales, sería muy difícil para los productores ecuatorianos competir en el mercado regional

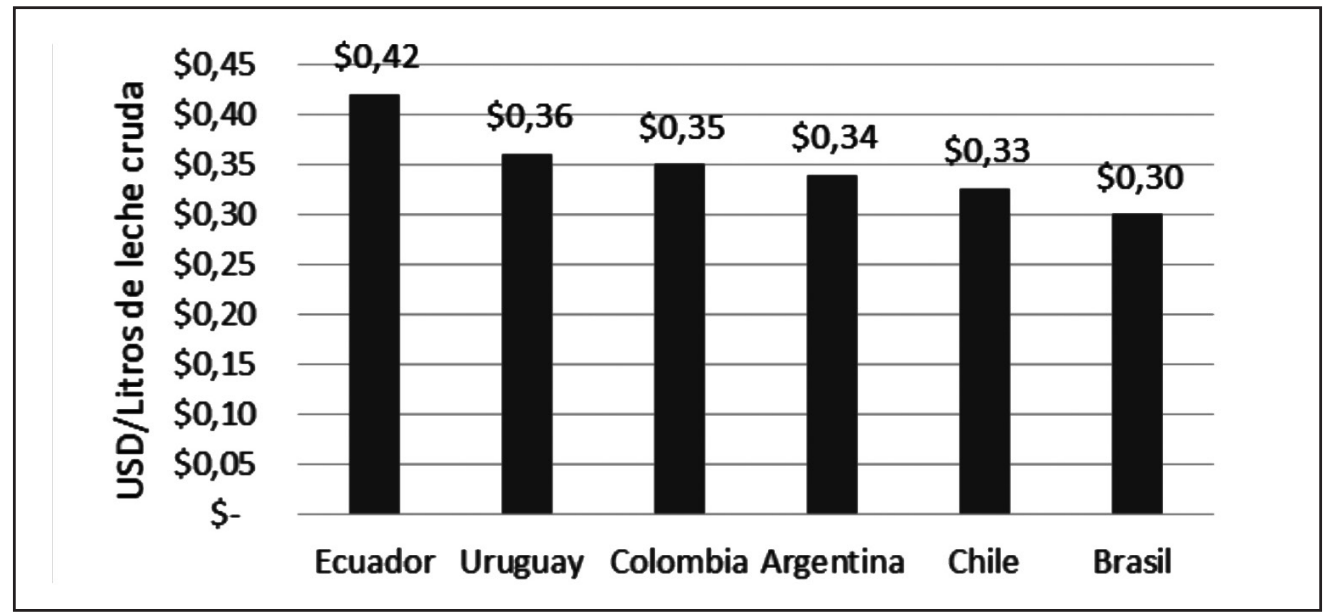

Figura 4. Precio de litro de leche al productor.

Fuente: Elaboración propia del autor con datos de la Federación de Ganaderos (FEDEGAN).

\section{Costos de producción}

De los datos presentados hasta aquí, se extrae que una alternativa para incrementar la competitividad de la leche producida en Ecuador en los mercados internacionales sería reducir el precio de la leche cruda que percibe el ganadero, sin embargo, una política de esta naturaleza afectaría a un gran número de pro- ductores, por lo que es necesario abrir un debate en el que intervengan todos los actores de la cadena de producción de leche en el país. En principio es preciso analizar los costos de producción por litro de leche a nivel local. Los rubros que mayor incidencia tienen sobre sus costos de producción son: balanceados y sales minerales con el $22 \%$, personal de finca con el $17 \%$ y fertilizantes y semillas con el 15\% (Figura 5).

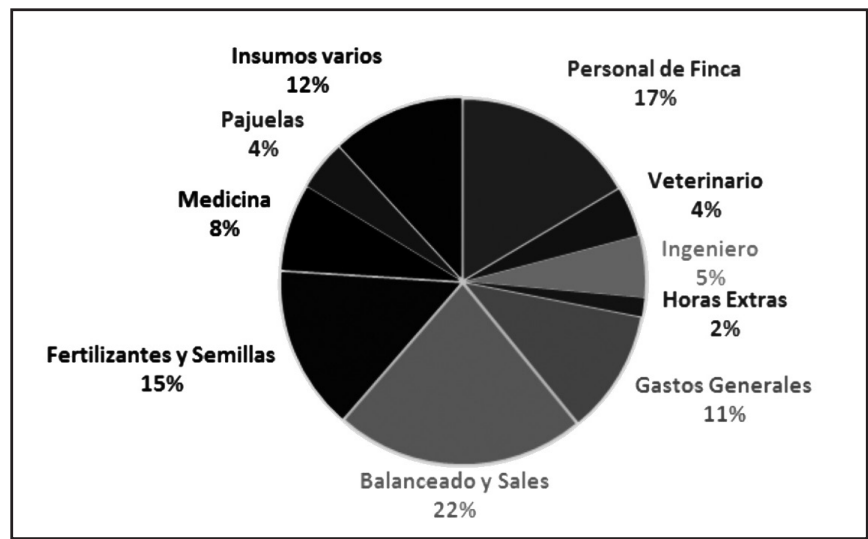

Figura 5. Distribución de los costos de producción de una ganadería de leche. 
La alimentación de los animales es el mayor rubro que tienen en las ganaderías, si se suma el balanceado y sales minerales $(22 \%)$ y fertilizantes y semillas (15\%) las dos equivalen al $37 \%$ de los costos de producción. El personal de finca representa el $17 \%$, en el país se considera que una ganadería con una extensión entre 30 y 40 hectáreas puede ser manejada por 3 a 4 persona.

\section{Conclusiones}

El ser declarado como país libre de fiebre aftosa por la OIE, luego de varias décadas de planes de vacunación, representa una gran meta alcanzada para el país, y una oportunidad de crecimiento y expansión a mercados internacionales para los ganaderos. La producción de leche en el país ha aumentado debido a diversos factores como la fijación del precio por litro de leche cruda y la esta-

\section{Referencias}

FEDEGAN. (2015). Precio de la Leche en mercado Internacional. Bogotá - Colombia: Federación de Ganaderos.

INEC. (2012). Encuesta de Producción y Superficie Agropecuaria. INEC.

Londinsky, A. (2015). América Latina y su potencial como productora de alimentos, el caso de los lácteos. (págs. 1-9). Federacion Panamericana de Lechería.

MAGAP. (2013). Acuerdo Ministerial 394. Ministerio de Agricultura Ganaderia y Pesca, 1-10. bilidad de ingresos de la leche en comparación con la producción de cultivos. Sin embargo, el consumo per cápita a nivel nacional es bajo (100 litros/ habitante/año) en comparación con otros países de la región. Esto ha provocado un desbalance de la relación entre oferta y demanda en ciertos meses del año (desde junio hasta septiembre) en los que existe sobreproducción de leche. El precio base que se paga por litro de leche (US \$ 0.42 ) no resulta competitivo a nivel regional. Países exportadores como Argentina y Brasil pagan a sus productores precios de entre US $\$ 0.35$ y US $\$ 0.30$ por litro, respectivamente. El costo más alto para los productores es la alimentación de los animales, el cual representa el $37 \%$ de los costos totales de producción. En este sentido, es necesario un debate sobre cómo reducir estos costos para poder ser más competitivos a nivel internacional y poder exportar los excedentes.

MAGAP. (2015). Ecuador recibe certificados de país libre de fiebre aftosa. Obtenido de: http:// www.agricultura.gob.ec/ecuador-recibe-certificados-de-pais-libre-de-fiebre-aftosa/

OIE. (2015). Mapa del Status Mundial de Fiebre Aftosa Mundial de los paises miembros de la OID. Obtenido de: http://www.oie.int/es/sanidad-animal-en-el-mundo/estatus-sanitario-oficial/fiebre-aftosa/es-fmd-carte/

Sharma, S. \& Rou, Z. (2014). China's Dairy Dilemma The Evolution and Future Trends of China's Dairy Industry. Washington D.C.: Institute for Agriculture and Trade Policy. 